\title{
Perspectives d'alternatives thérapeutiques antimicrobiennes aux antibiotiques en élevage
}

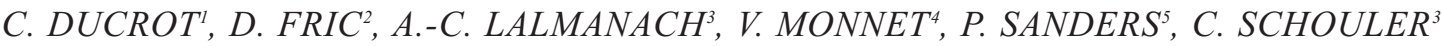 \\ 'Épidémiologie Animale, INRA, 63122, Saint-Genès-Champanelle, France \\ 2 Vétérinaire praticien homéopathe, Commission élevage ITAB, 23150, Sous-Parsat, France \\ ${ }^{3}$ ISP, INRA, Université François Rabelais de Tours, 37380, Nouzilly, France \\ ${ }^{4}$ Micalis Institute, INRA, AgroParisTech, Université Paris-Saclay, 78350, Jouy-en-Josas, France \\ ${ }^{5}$ Anses, Laboratoire de Fougères, Bâtiment Bioagropolis, 35306, Fougères, France \\ Courriel : christian.ducrot@inra.fr
}

La diminution drastique de l'usage des antibiotiques en élevage passe par la prévention des maladies et les mesures de biosécurité, l'usage raisonné des antibiotiques, mais également par le développement de méthodes alternatives ou complémentaires à l'antibiothérapie. Différentes approches sont étudiées dont certaines sont basées sur des découvertes biologiques anciennes, insuffisamment explorées et qui sont remises au goût du jour.

Les avancées médicales majeures dans le traitement des maladies infectieuses sont largement dues à la découverte et au développement des antibiotiques depuis les travaux pionniers sur la pénicilline découverte en 1928. L'âge d'or des antibiotiques (années 1940-1960) durant lequel la majorité des familles ont été identifiées a été suivi d'une utilisation très étendue et souvent inappropriée (Carlet et le Coz 2015), et a engendré l'apparition de résistances bactériennes simples puis multiples qui sont aujourd'hui une menace tangible d'échec thérapeutique de la médecine moderne (Zahar et Lesprit 2014). Cette menace est aggravée par le déclin de l'identification et du développement de nouveaux antibiotiques en ce début de XXIème siècle (Donadio et al 2010). Aussi, « le risque de se trouver, à moyenne échéance, dans une véritable impasse thérapeutique empêchant de traiter certaines infections microbiennes est donc majeur, y compris dans les pays développés, à moins que des actions de lutte ambitieuses et coordonnées soient mises en place » (European Commission 2011). Cette préoccupation a conduit les instances internationales (WHO, FAO et OIE 2010, OMS 2015) à adopter des plans concertés pour optimiser l'usage des antibiotiques et promouvoir la recherche pour des solutions alternatives, tant chez l'Homme que chez l'animal. Les pouvoirs publics nationaux et européens (European Commission 2011) ont eux aussi engagé des actions ambitieuses. En santé animale en France, le plan EcoAntibio 2017 (Ministère de l'agriculture 2012) comprend un ensemble de 40 mesures complémentaires destinées à promouvoir des actions préventives des maladies, mettre en œuvre un usage raisonné et économe des antibiotiques et développer des approches thérapeutiques alternatives (disposition numéro 19).

En élevage, une réduction drastique de l'usage des antibiotiques repose sur trois approches qui devraient être poursuivies de manière complémentaire : i) Mettre l'accent sur la prévention des maladies infectieuses, ii) rationaliser et optimiser l'usage des antibiotiques en cas de maladie infectieuse, et iii) utiliser des traitements curatifs alternatifs aux antibiotiques. Cet article aborde le troisième point, en mettant l'accent sur des approches thérapeutiques dont l'usage n'est pas réglementé de manière satisfaisante actuellement ou qui pourraient représenter un potentiel dans le futur. Un dossier récent (Le point vétérinaire 2016) aborde un ensemble d'approches thérapeutiques déjà existantes et utilisées en pratique sur le terrain. Ainsi, les bases et les circonstances d'utilisation sont abordées notamment pour l'homéopathie (Ansoud 2016, Dordain 2016b, Filliat et Souvestre 2016, Issautier 2016), la phytothérapie (Dordain 2016a, Filliat et Souvestre 2016, Mauvisseau 2016), l'acupuncture (Lecat 2016), ces approches étant comparées (Gromont 2016a) et mises en perspective dans une autre approche de la santé du troupeau (Gromont 2016b). Il existe aussi d'autres possibilités thérapeutiques sur lesquelles des travaux de recherche et de développement sont en cours. Le présent article présente plusieurs d'entre elles, leurs caractéristiques, leurs avantages et inconvénients, leurs contraintes techniques et réglementaires, leurs enjeux et perspectives. Elles sont présentées en fonction de la plausibilité plus ou moins lointaine d'être mises en œuvre en pratique.

\section{1 / Phytothérapie}

\section{1 / Pharmacopée}

Il paraît paradoxal d'aborder ici la phytothérapie comme médicament en développement dans la mesure où l'homme a toujours eu recours à l'utilisation des plantes pour se soigner ou pour soigner les animaux et les remèdes à base de plantes ou de plantes transformées existent depuis la plus haute antiquité. Néanmoins, il existe un vide juridique partiel et l'utilisation thérapeutique de la phytothérapie ne va pas de soi dans le contexte actuel où l'autorisation de mise sur le marché d'un médicament requiert d'assurer la preuve de son innocuité, de son efficacité et de l'absence de résidus dans les produits issus des animaux traités, éléments dont on ne dispose pas précisément.

La phytothérapie désigne l'utilisation des plantes à des fins thérapeutiques. 
Selon les cas, la plante entière ou certaines parties de celle-ci sont utilisées, incluant les parties souterraines, tiges, feuilles, fleurs, sommités fleuries, bourgeons, fruits, graines, qui sont utilisées sous forme fraiches ou séchées. Plusieurs types de transformation de ces plantes sont appliqués : infusion, décoction (dans l'eau maintenue bouillante), macération (dans l'eau froide), extraction hydroalcoolique, pour obtenir différentes préparations finales. L'aromathérapie, qui est l'utilisation médicale des extraits aromatiques de plantes (essences et huiles essentielles) est une des modalités de la phytothérapie. Les propriétés thérapeutiques des plantes sont associées à la présence en leur sein de molécules appartenant à une grande diversité de classes chimiques (par exemple alcaloïdes, terpènes...).

Pour leur utilisation chez l'Homme, une liste de drogues végétales a été révisée en 2012 dans la pharmacopée française, scindée en deux parties. La partie A correspond aux 364 drogues végétales à base de plantes médicinales utilisées traditionnellement; les pharmaciens ont le monopole de la vente de ces plantes médicinales mais 148 d'entre elles peuvent être vendues hors circuit pharmaceutique car utilisées dans des produits cosmétiques ou alimentaires. La partie $\mathrm{B}$ comprend 123 drogues végétales à base de plantes médicinales utilisées traditionnellement en l'état ou sous forme de préparations et dont les effets indésirables potentiels sont supérieurs au bénéfice thérapeutique attendu ; ces plantes peuvent toutefois servir à la préparation de médicaments homéopathiques.

L'agence européenne du médicament a mis en place une démarche de recensement et d'évaluation des plantes à vertus thérapeutiques dans le cadre de la directive 2004/24/CE. Le recours à des plantes dites médicinales existe dans toutes les cultures. L'étude de ces pharmacopées ancestrales (par exemple, chinoise, ayurvédique (Inde), amazone) constitue un domaine de recherche pluridisciplinaire très actif dans l'identification de nouveaux principes actifs. Dans les 40 dernières années, moins de $40 \%$ des petites molécules développées en tant que médicament étaient d'origine synthétique, plus de la moitié étaient dérivées ou inspirées de la nature (Newman et al 2012).

\section{2 / Traitement des maladies infectieuses}

Les plantes contiennent un très grand nombre de molécules pouvant avoir des modes d'action pharmacologiques variés voire synergiques vis-à-vis des bactéries ou de l'animal infecté, en prévention ou en traitement des maladies d'étiologie bactérienne. Chez les bactéries, l'activité peut être antibactérienne ou réduire la virulence des bactéries en perturbant, par exemple, la communication bactérienne ou la formation de biofilm. Chez l'animal, les propriétés des plantes peuvent être anti-inflammatoires, immunomodulatrices ou physiologiques, réduisant les signes cliniques de l'infection tout en aidant aux processus de guérison.

À titre d'exemples dans le cadre de maladies infectieuses, certaines plantes comme l'eucalyptus, le fenouil, la muscade ont des effets antibiotiques. D'autres contiennent des tannins qui ont un effet préventif ou curatif pour les diarrhées, par exemple l'épicéa ou le chêne. De nombreuses revues bibliographiques discutent des propriétés antibactériennes de substances (par exemple le carvacrol ou le thymol) présentes dans les huiles essentielles. Ces effets observés in vitro ne sont pas forcément reproduits directement au niveau de l'intestin du fait des propriétés physico-chimiques de ces molécules. L'obtention de l'effet, au niveau distal de l'intestin, nécessite une formulation galénique libérant les molécules actives à ce niveau (de Lange 2010). En aviculture, les huiles essentielles et les tannins peuvent être utilisables pour contrôler l'entérite nécrotique (Diaz-Carrasco 2016). Chez le poisson, l'utilisation de la phytothérapie avec différents extraits et huiles essentielles de nombreuses plantes (par exemple, ail, romarin, curcuma, origan, romarin) contenant des composés phénoliques, polysaccharides, protéoglycanes, flavonoïdes fait l'objet de multiples travaux pour accroître la résistance des poissons aux infections bactériennes (Bulfon et al 2015).

Par ailleurs, dans un dossier consacré aux thérapeutiques alternatives (Le point vétérinaire 2016), plusieurs illustrations sont données. Un article traite de la phytothérapie en élevage bovin, avec des applications aux troubles infectieux diarrhéiques et pulmonaires (Dordain 2016a). Un autre aborde son usage en médecine collective, avec l'exemple de l'action sur l'équilibre microbien et parasitaire du tube digestif chez les volailles (Filliat et Souvestre 2016). L'usage des huiles essentielles contre les maladies bactériennes est aussi abordé à partir du « phytogramme ${ }^{\circledR}$ » (Mauvisseau 2016), pour identifier lesquelles sont les plus inhibitrices de la multiplication de la souche bactérienne concernée, avec des applications illustrées sur la pathologie digestive, respiratoire et mammaire.

\section{3 / Contraintes réglementaires}

Les procédures d'évaluation préalable à l'autorisation de mise sur le marché nécessitent de garantir la qualité du pro- duit notamment la reproductibilité de sa composition quantitative et qualitative d'un lot de médicament à l'autre. C'est un premier défi pour des produits de phytothérapie dont la composition de la matière première (plante) peut varier en fonction de nombreux facteurs (origine, conditions de cultures...) et s'avérer complexe compte tenu de la diversité des molécules présentes. Le second défi est la démonstration de l'efficacité thérapeutique. Elle est basée sur la connaissance des mécanismes d'action et sur la démonstration de l'efficacité en conditions de terrain.

Pour l'Homme, le choix a été fait au niveau européen de se baser sur les pharmacopées traditionnelles nationales en mettant en place une démarche d'évaluation spécifique à la phytothérapie. Pour la médecine vétérinaire, la preuve de l'efficacité de la phytothérapie est à apporter en fonction de l'indication thérapeutique revendiquée. La démonstration de l'efficacité préventive et/ou curative d'un traitement de phytothérapie, alternatif à un traitement antibiotique, nécessite donc des essais cliniques ; une alternative proposée par l'Agence nationale de sécurité sanitaire de l'alimentation, de l'environnement et du travail (Anses 2016) est d'apporter la preuve d'un usage bien établi depuis plus de 10 ans dans la communauté européenne.

En médecine vétérinaire, le cadre réglementaire prévoit aussi d'assurer la protection de la santé publique, notamment pour limiter l'exposition des consommateurs à des résidus nocifs (Règlement européen 470/2009). Dans ce cadre, plus d'une centaine de plantes (sous forme d'huiles essentielles, ou partie de plantes) ont été évaluées pour une utilisation sous forme de médicaments vétérinaires chez les animaux producteurs de denrées alimentaires sans nécessité de fixer de limites maximales de résidus (Anses 2016). La moitié environ de ces plantes est autorisée dans la composition de médicaments vétérinaires (tableau $\mathrm{A}$ du règlement $37 / 2010$ ), uniquement en tant que médicaments vétérinaires homéopathiques ou topiques (usage externe local). L'autre moitié peut être utilisée chez toutes les espèces, avec cependant quelques restrictions d'usage (espèces animales, âge) ; la plupart de ces plantes autorisées font partie du régime alimentaire de l'Homme et des animaux.

Bien que la réglementation européenne sur les productions biologiques encourage le recours à des produits de phytothérapie, très peu de ces produits sont mis sur le marché en tant que médicaments vétérinaires (Mayer et al 2014) et le cadre réglementaire du médicament vétérinaire semble un frein à leur développement. En France, le décret n²013-752 
permet un allègement des pièces du dossier de demande d'Autorisation de Mise sur le Marché (AMM) des médicaments vétérinaires à base de plantes. L'avis de l'Anses de février 2016 (Anses 2016) recommande d'établir une liste prioritaire de substances végétales aujourd'hui nécessaires pour la phytothérapie des animaux producteurs de denrées afin d'encourager leur évaluation dans le cadre réglementaire ; il propose aussi des voies d'allégements sur l'évaluation de la qualité et la démonstration de l'efficacité.

\section{4 / Conditions d'utilisation actuelles}

Les plantes et produits dérivés ont des propriétés nutritionnelles et métaboliques qui peuvent conduire à une combinaison d'effets physiologiques et pharmacologiques, sachant qu'il n'y a pas de frontière claire entre un état d'équilibre et l'apparition d'un état pathologique. La difficulté avec les plantes médicinales ou produits dérivés est leur positionnement sur des statuts réglementaires différents, entre les secteurs de l'alimentation et de la supplémentation alimentaire et la revendication de propriétés ou indications thérapeutiques, domaine du médicament vétérinaire.

Compte tenu de la définition juridique actuelle du médicament vétérinaire (directive 2001/82/CE) dès lors que le produit à base de plantes est positionné sur des indications de prévention et de traitement des maladies infectieuses avec une présentation destinée à une administration à l'animal, il relève par fonction et par présentation du statut de médicament vétérinaire et doit se conformer à cette réglementation et suivre le processus d'évaluation conduisant à une AMM.

Dans le cadre de la cascade (article L 5143-4 du Code de la Santé Publique) qui détermine les conditions de prescription en l'absence de médicament vétérinaire approprié autorisé, le vétérinaire peut également prescrire une préparation magistrale à base de drogues végétales et produits dérivés, qui sera fabriquée par le pharmacien à partir de matières premières.

Le produit à base de plantes peut aussi être positionné dans certains cas comme un additif à l'alimentation animale, et ajouté aux aliments pour animaux ou à l'eau pour remplir des fonctions définies par le règlement européen (CE) 767/2009 comme ayant un effet positif sur la production, le rendement ou le bien-être, en influençant la flore gastro-intestinale ou la digestibilité des aliments pour animaux. La réglementation en matière d'alimentation animale doit alors être respectée.
Enfin, il y a consensus sur la nécessité d'adapter les cadres réglementaires pour clarifier le positionnement des produits à base de plantes en tant que produits utilisables en production animale conduisant à un moindre recours aux antibiotiques.

\section{2 / Phagothérapie}

\section{1 / Phages}

La phagothérapie est une thérapie vieille d'un siècle qui revient en force sur le devant de la scène ainsi que le reflète l'augmentation du nombre de publications sur le sujet depuis les années 2000. Elle fait appel à l'utilisation de phages virulents comme agents antibactériens. Les phages, ou bactériophages, sont des virus n'infectant que des bactéries, constitués d'une enveloppe protéique (la capside) qui contient de l'ADN (dans la majorité des cas) ou de l'ARN. Ils présentent une grande variété tant au niveau morphologique qu'au niveau génomique (figure 1). Ainsi, certains phages possèdent une queue pouvant être rigide ou contractile (figure 2), d'autres des appendices, des manchons, des spicules caudales. Ils représenteraient l'entité biologique la plus abondante de la biosphère. Ils interviennent dans le maintien de la diversité et la régulation des populations bactériennes au sein des écosystèmes.

Le cycle de vie des phages peut être de deux types (figure 3) : i) soit lysogénique, avec intégration de l'ADN du phage dans le chromosome de la bacté- rie ; ii) soit virulent, avec multiplication du phage dans la bactérie, destruction de la bactérie et libération de nouveaux phages. Certains phages ne se répliquent que suivant un cycle virulent et sont nommés phages virulents, d'autres peuvent se répliquer selon les deux cycles et sont nommés phages tempérés. La caractéristique principale du cycle lysogénique est que le phage ne détruit pas la bactérie et va ainsi pouvoir se maintenir à l'état dormant au sein de la population bactérienne.

\section{2 / Thérapie phagique}

Seuls les phages se multipliant selon un cycle lytique sont considérés en thérapeutique car ils peuvent agir en détruisant les bactéries. Divers points sont à prendre en considération pour maximiser les chances de succès de la thérapie phagique (Carlton 1999, Sulakvelidze et al 2001, Inal 2003, Loc-Carrillo et Abedon 2011, Prevel et Dufour 2016). Le plus important est de s'assurer que les phages utilisés sont strictement à cycle lytique et que leur génome ne code pas de gènes indésirables comme des gènes de virulence. Bien que plusieurs études rapportent que les phages pourraient coder des gènes de résistance aux antibiotiques, une étude récente tend à démontrer le contraire (Enault et al 2016). Les phages ont une étroite spécificité d'hôtes dans la mesure où ils n'agissent que sur certaines souches bactériennes. Avant traitement il est donc préférable d'isoler la bactérie responsable de l'infection pour pouvoir cibler le traitement par les phages. Ensuite deux types d'approches thérapeutiques peuvent être envisagées : $i$ ) soit

Figure 1. Classification des phages selon Ackermann (Ackermann 2003).

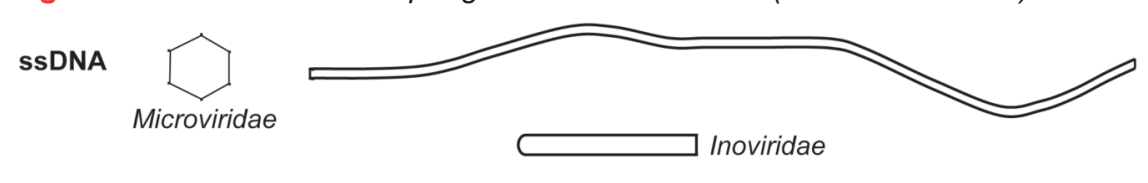

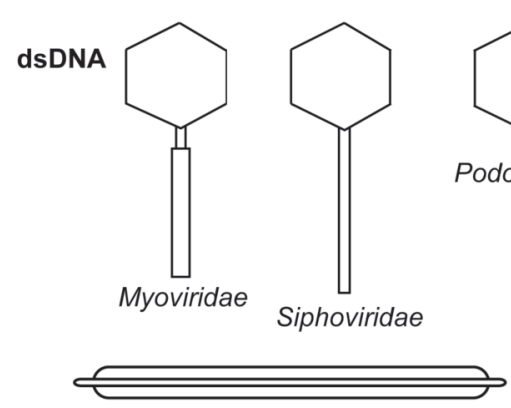

Lipothrixviridae

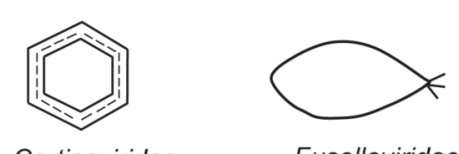

Corticoviridae

Fuselloviridae
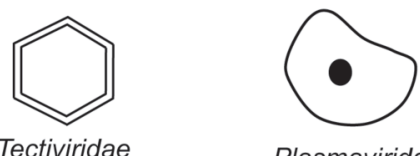

Plasmaviridae

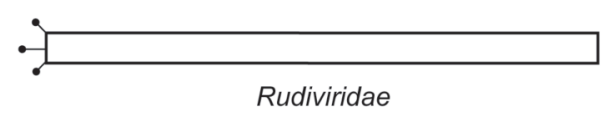

dsRNA

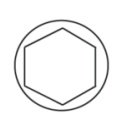

Cystoviridae 
une stratégie de traitement « prêt-àporter » avec l'administration de cocktails de bactériophages ciblant les principales souches bactériennes de l'espèce identifiée, cette approche nécessitant dans l'idéal une veille microbiologique pour adapter régulièrement la composition de ces cocktails ; ii) soit une stratégie de traitement « sur mesure » avec l'administration d'un cocktail de bactériophages ciblant spécifiquement la souche bactérienne responsable (Pirnay et al 2011). Il est primordial de s'assurer de l'extrême pureté des suspensions phagiques utilisées afin d'éviter la présence de débris bactériens, de bactéries, de phages lysogènes ou de toxines.

\section{3 / Enjeux et questions de recherche}

Il est dans un premier temps essentiel de développer des projets de recherche sur l'évaluation du potentiel thérapeutique de la phagothérapie en alternative ou en complément à l'antibiothérapie. Ces projets devraient conduire à étudier la biologie des phages, leurs effets sur l'organisme, leur impact potentiel sur les écosystèmes animaux et environnementaux, l'apparition de systèmes de résistance aux phages et l'évaluation de l'immunité adaptative des organismes traités qui pourrait impacter l'efficacité de traitements successifs. Ils devraient viser à produire des suspensions phagiques répondant aux normes GMP (« Good Manufacturing Pratice ») afin de les valider en essais cliniques vétérinaires sur la base d'une liste de critères (Pirnay et al 2015).

\section{4 / Perspectives d'utilisation}

Une question préalable majeure est de savoir si on doit réserver ou non l'usage de la phagothérapie à la médecine humaine comme traitement de dernier recours pour des patients en impasse thérapeutique face à des bactéries résistantes aux antibiotiques. En effet, si la thérapie phagique est appliquée en médecine vétérinaire, deux types d'intervention peuvent être envisagés : au niveau de l'individu ou à l'échelle du lot ou du troupeau en métaphylaxie. Dans ce dernier cas plus particulièrement, il est indispensable d'évaluer préalablement quelles pourraient être les conséquences du relargage massif de phages dans l'environnement, car les phages sont résistants dans le milieu extérieur et pourraient tolérer des conditions physico-chimiques difficiles. De ce fait il est important d'évaluer leur impact sur la modulation éventuelle des écosystèmes en participant à la sélection de souches résistantes aux phages, souches qui pourraient alors infecter l'Homme et engendrer de nouvelles impasses thérapeutiques. Le débat
Figure 2. Coliphage ESCO3 observé par coloration négative à l'acétate d'uranyle en microscopie électronique.

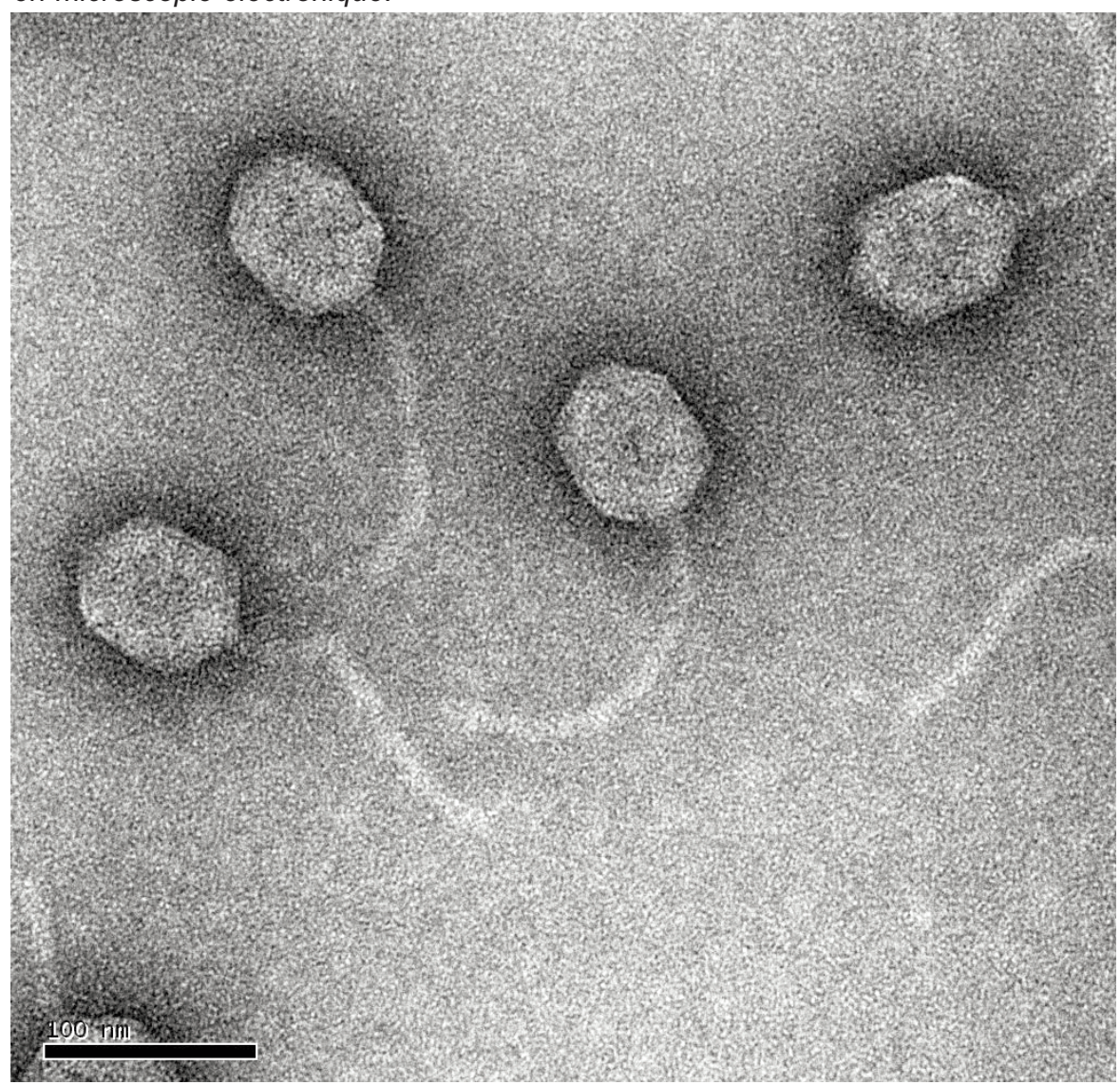

Figure 3. Cycles de réplication des phages (http://www.microbiologie-medicale.fr/virologie/generalitesvirus.htm).

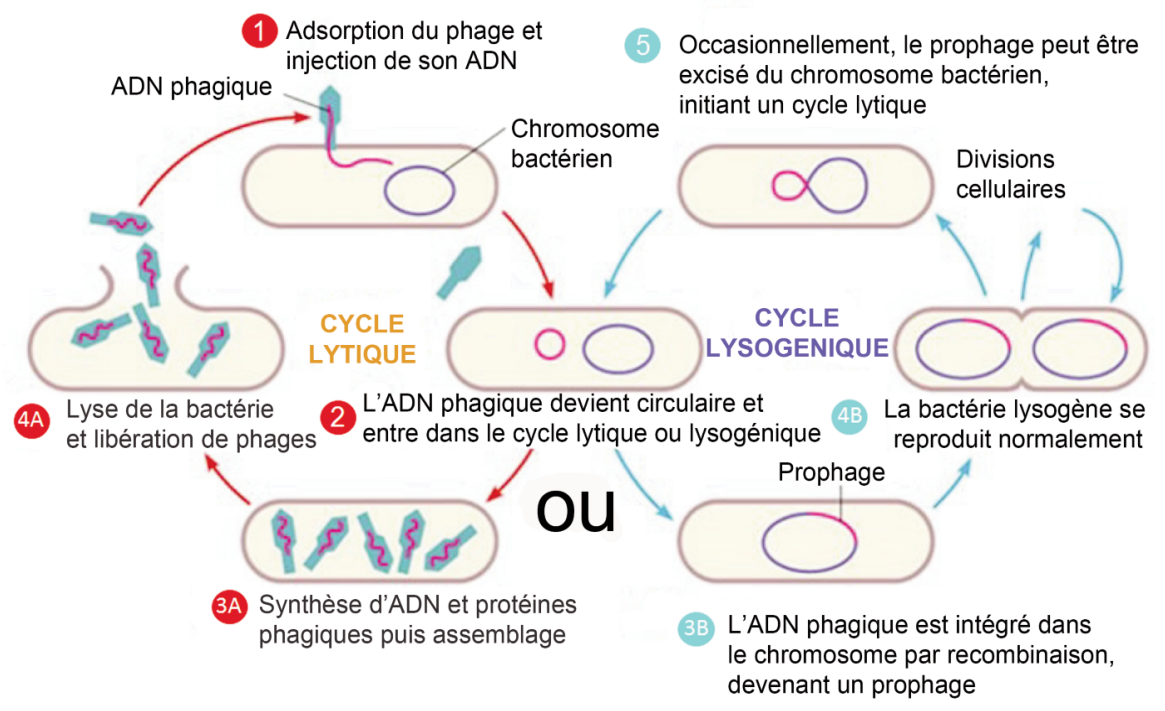

sur l'utilisation massive des phages en médecine vétérinaire est donc ouvert et la modélisation des risques potentiels est essentielle. Toutefois, une application dérivée des phages serait l'utilisation d'enzymes phagiques, produites par les phages, telles que les endolysines qui dégradent le peptidoglycane et les dépolymérases qui dégradent les polysaccharides capsulaires (Pires et al 2016). De telles enzymes couplées aux antibiotiques pourraient permettre de déstructurer les biofilms bactériens qui sont source d'agents pathogènes et qui sont très présents dans les bâtiments d'élevage, notamment dans les conduites d'eau.

\section{5 / Contraintes réglementaires}

Aux États-Unis, la « Food and Drug Administration » (FDA) a autorisé des produits à base de phages pour un usage de décontamination en industrie agroalimentaire, toutefois elle n'a pas pour le 
moment autorisé un usage en médecine. En France, les phages ne possèdent plus d'AMM en médecine humaine et vétérinaire. L'application de la réglementation relative aux médicaments n'est pas totalement adaptée au développement industriel de phages ce qui entraine un flou juridique et législatif. Un positionnement sur l'utilisation des phages en tant qu'agent thérapeutique et bio-médicament doit être établi. Ce travail serait en cours d'élaboration par l'Agence Nationale de Sécurité du Médicament (ANSM) (JO Sénat du 22/05/2013). À l'heure actuelle, la phagothérapie n'est appliquée que très rarement en médecine humaine, dans des cas d'impasses thérapeutiques (usage dit compassionnel dans le cadre de l'article 37 de la déclaration d'Helsinki).

\section{3 / Peptides antimicrobiens ou peptides de défense de l'hôte}

\section{1 / Peptides de défense de l'hôte}

Découverts dans les années 1970 à partir d'études sur la peau des amphibiens et sur les insectes (figure 4), les peptides antimicrobiens sont des molécules de défense produites par l'organisme. Ils ont été renommés depuis une dizaine d'années comme peptides de défense de l'hôte (HDP) car leur activité biologique s'étend bien au-delà d'une fonction antimicrobienne stricte (Hancock et al 2016). Ils sont présents dans virtuellement toutes les formes de vie du royaume bactérien, fongique, animal et végétal, et sont des composants essentiels très anciens de l'immunité innée. L'« Antimicrobial Peptide Database " (http:// aps.unmc.edu/AP/main.php) contient aujourd'hui plus de 2700 peptides répertoriés dont plus de 2000 d'origine animale. Chez les animaux, les HDP sont produits essentiellement par les cellules épithéliales et les phagocytes situés à l'interface entre l'organisme et son environnement (peau, tractus respiratoire, digestif, urinaire et reproducteur).

Les HDP sont de petite taille (12 à 50 acides aminés), cationiques et à domaines hydrophobes (Zasloff 2002), ce qui explique leur propension à interagir avec les membranes bactériennes. Leur classification en quatre classes est basée sur leur structure secondaire avec deux principales chez les animaux : peptides en hélice-a (cathélicidines) et peptides en feuillet-b (défensines), ces derniers étant les plus abondants et les plus compacts. Chaque classe peut contenir de nombreux variants assurant à l'hôte un répertoire diversifié de peptides à large spectre antibactérien.

Figure 4. Études pionnières des principaux peptides antimicrobiens (Courtoisie du Dr Mohamed Amiche, Directeur Adjoint du Groupe de Recherche (GdR3625 CNRS) MultiFonction des Peptides AntiMicrobiens (MuFoPAM)).

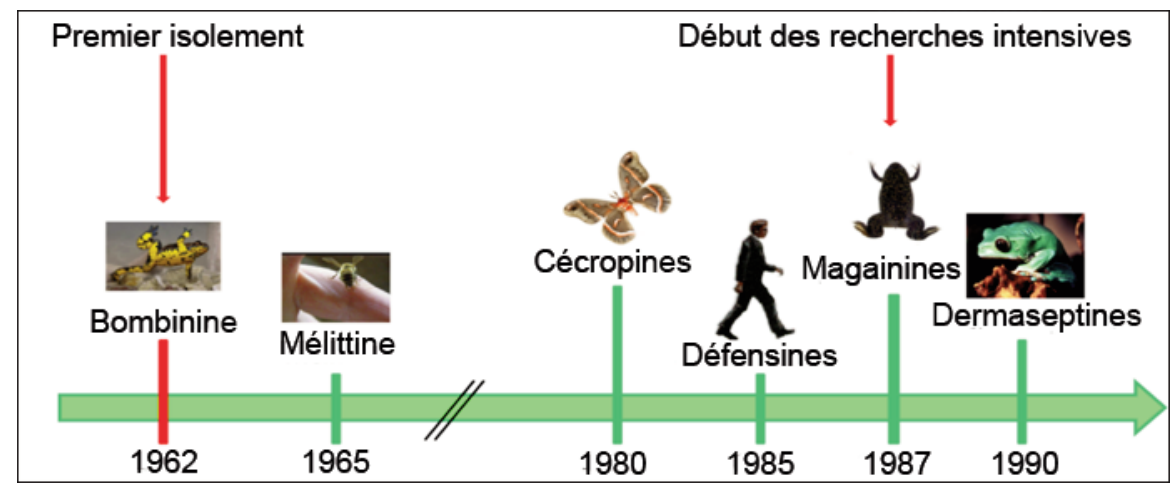

\section{2 / Activité biologique}

L'activité biologique des HDP est d'abord décrite comme antimicrobienne compte tenu de leur capacité à déstabiliser les membranes des microbes jusqu'à les rompre. Certains peuvent pénétrer à l'intérieur des microbes pour perturber la machinerie cellulaire (réplication des acides nucléiques, transcription, traduction). Ces mécanismes de perturbation sans haute affinité pour une cible microbienne spécifique réduisent la possibilité pour les bactéries de développer des résistances à ces peptides. Cependant, l'activité antimicrobienne est souvent diminuée en conditions physiologiques dans la mesure où des inhibiteurs sont présents. Toutefois, à proximité de la source de production des HDP, les concentrations atteintes sont suffisamment importantes pour exercer une activité de barrière aux pathogènes au niveau des muqueuses. Par ailleurs, à des concentrations inférieures aux concentrations inhibitrices sur les bactéries, certains HDP peuvent aussi prévenir la formation de biofilms, source de colonisation bactérienne persistante et d'infections chroniques.

Depuis les années 2000, des études ont démontré que des HDP des animaux et de l'Homme peuvent recruter des cellules immunitaires et les stimuler, ceci à des concentrations en HDP bien inférieures à celles requises pour une activité antimicrobienne directe. Depuis, il a été montré que les HDP ont non seulement des propriétés immuno-modulatrices mais aussi une action anti-inflammatoire, anti-endotoxine, anti-tumorale, pro-angiogenèse et sur la réparation des lésions.

\section{3 / Enjeux et questions de recherche}

Les multiples fonctions des HDP issus des animaux, à la fois directes sur les microbes eux-mêmes et indirectes en promouvant l'immunité de l'hôte qui les héberge, constituent un avantage majeur qui mérite d'être approfondi. Dans ce sens, l'étude de l'apport des HDP comme adjuvants lors d'une vaccination est à explorer. Par ailleurs, l'utilisation thérapeutique en synergie avec d'autres antimicrobiens pour augmenter l'efficacité et diminuer le risque d'apparition de résistances bactériennes est également une voie prometteuse. Toutefois, leur effet, qui peut être délétère sur les cellules de l'organisme et sur le microbiote, reste également à mesurer pour évaluer le risque de toxicité et de déséquilibre du microbiote. Stimuler la production des peptides antimicrobiens propres à l'organisme hôte (grâce à des additifs alimentaires, médicaments) est une autre voie prometteuse qui commence à être abordée ces dernières années en recherche (Prado Montes de Oca 2013).

\section{4 / Contraintes à l'usage des peptides antimicrobiens}

Le processus de développement de drogues pharmaceutiques depuis leur découverte jusqu'à leur mise sur le marché est long (plus de 10 ans) et coûteux, avec de nombreuses contraintes et un taux de succès très bas pour les antimicrobiens (Projan et Shlaes 2004). On recense aujourd'hui moins d'une vingtaine de peptides antimicrobiens en essai clinique pour la médecine humaine, avec des applications antimicrobiennes et anti-inflammatoires dermatologiques et plus rarement systémique. En médecine vétérinaire, les coûts de traitement doivent être considérablement inférieurs aux coûts pratiqués en médecine humaine pour une commercialisation possible, ce qui constitue un frein majeur à l'apparition de nouveaux traitements anti-infectieux en élevage.

La complexité structurale des molécules naturelles les rend souvent difficiles à obtenir par voie recombinante qui est la plus facilement transposable à l'échelle industrielle. La synthèse chimique est également compliquée et coûteuse pour un développement pharmaceutique vétérinaire; de plus, le choix de synthétiser 
par voie chimique des peptides raccourcis à la longueur strictement active peut diminuer la stabilité, la biodisponibilité et entraîner des effets secondaires de toxicité in vivo. Enfin, l'industrie pharmaceutique n'est pas encline à utiliser des produits animaux (sous-produits des filières viande) qui pourraient constituer pourtant une source potentielle d'HDP à extraire et à purifier à moindre coût.

\section{5 / Perspectives d'utilisation}

Les HDP des animaux sont des molécules naturelles qui présentent donc l'avantage d'être bien tolérées par les organismes et plus facilement acceptables d'un point de vue sociétal. Si nous n'avons pas actuellement de perspectives d'usage en pratique de ces HDP à court et moyen terme, leur large spectre antibactérien allié à leur efficacité vis-à-vis de souches multi-résistantes aux antibiotiques ainsi que leur effet promouvant l'immunité de l'hôte laissent toutefois de bonnes raisons d'espérer à l'avenir de nouveaux traitements utilisant des HDP pour lutter contre les maladies infectieuses majeures et émergentes en élevage.

\section{4 / «Quorum quenching » et « quorum sensing »}

\section{1 / Conversations bactériennes}

Dans les années 1960, deux études pionnières ont montré que les bactéries n'étaient pas indépendantes les unes des autres mais qu'elles étaient capables de communiquer entre elles. Depuis, de nombreuses études ont montré que cette communication était la norme dans le monde bactérien, introduisant la notion de " conversations bactériennes » (Monnet et al 2016). En coordonnant l'expression de gènes au sein d'une population, cette communication donne du poids à un ensemble de bactéries qui modifient ainsi leur comportement de manière coordonnée au sein d'une même espèce où d'espèces proches partageant le même mode de communication.

Dans les grandes lignes, le scenario est toujours à peu près le même : les bactéries produisent et sécrètent des molécules de signalement qui indiquent leur présence et s'accumulent dans le milieu extracellulaire. Lorsque la concentration de ces molécules atteint une valeur seuil, d'où le nom de «quorum sensing » (détection d'atteinte du quorum), les molécules sont perçues par l'ensemble de la population bactérienne soit au niveau d'un récepteur à la surface bactérienne, soit par un transporteur qui « réinternalise » (réincorpore) la molécule de signalement dans la bactérie. Cette perception modifie l'expression de gènes cibles de manière coordonnée entre toutes les bactéries présentes qui ont réagi au signal, donnant ainsi un poids fort à la réponse et au changement de phénotype (forme ou action de la bactérie) (figure 5). La nature des molécules diffère selon qu'il s'agit de bactéries de type Gram+, comme par exemple Staphylococcus aureus, qui utilisent comme molécule de signalement des oligopeptides, ou Gram-, comme par exemple Pseudomonas aeruginosa, qui utilisent des acyl homoserine lactones. $\mathrm{Au}$-delà de ces deux groupes de molécules de signalement bien caractérisées, de nouvelles molécules sont constamment décrites.

De nombreuses fonctions sont contrôlées par des mécanismes de communication de type «quorum sensing » : conjugaison, transformation, sporulation, formation de biofilms et surtout virulence pour les bactéries pathogènes. Dès lors, il devient intéressant de chercher à contrôler cette communication soit pour induire certaines fonctions d'intérêt soit au contraire pour éviter l'expression de gènes impliqués dans la virulence. Dans ce dernier cas, on parle de « quorum quenching » (blocage du mécanisme de " quorum sensing »); il s'agit de chercher à interférer avec le mécanisme de «quo- rum sensing ". Cette approche vise à empêcher l'expression de gènes de virulence sans forcément éliminer les bactéries ce qui permet de limiter l'apparition de mécanismes de résistance comme ceux observés dans le cas de l'utilisation d'antibiotiques.

\section{2 / Enjeux et questions de recherche}

Le contrôle de la communication bactérienne permet d'empêcher l'expression de gènes de virulence. C'est donc une des pistes identifiées pour développer des alternatives aux antibiotiques. Une grande partie des travaux publiés dans ce domaine se base le plus souvent sur la connaissance des mécanismes de communication. Ils visent différentes étapes du mécanisme de "quorum sensing 》 (figure 6) :

\section{a) Production de la molécule de signalement}

La première cible est l'étape de synthèse de la molécule de signalement. Un article récent rapporte par exemple la conception par analyse structurale et mutagénèse dirigée d'un composé [(z)-5-octylidenethiazolidine-2, 4-dione] ayant une affinité pour le site actif de l'enzyme qui synthétise l'acyl homoserine lactone de Pseudomonas aeruginosa et qui s'avère être un inhibiteur de cette enzyme et donc de la communication cellulaire. Ce composé est proposé pour servir de base aux développements d'inhibiteurs d'autres acyl homoserine lactones (Lidor et al 2015).

b) Stabilité ou biodisponibilité de la molécule de signalement dans le milieu extracellulaire

Si la production de la molécule de signalement n'a pas été empêchée, des stratégies de piégeage ou de dégradation dans le milieu extérieur peuvent être envisagées. Des enzymes de type lactonases, capables de dégrader les acyl homoserine lactones des bactéries Gram- ont été identifiées et utilisées avec

Figure 5. Représentation schématique de la régulation d'expression de gènes par " quorum sensing ».

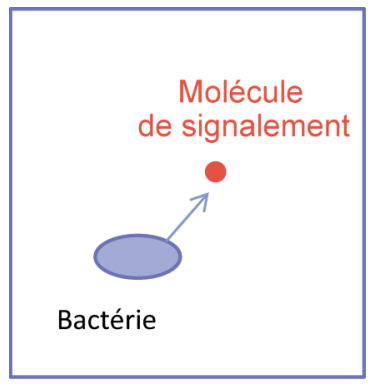

I. Les bactéries produisent des molécules de signalement

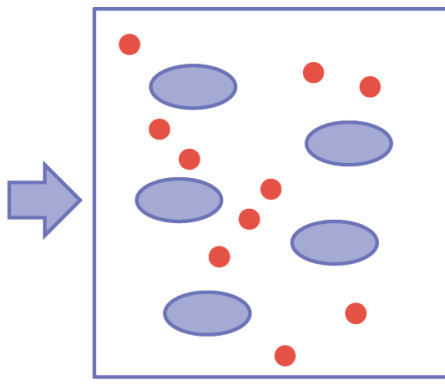

II. Les molécules de signalement s'accumulent lorsque la population bactérienne augmente

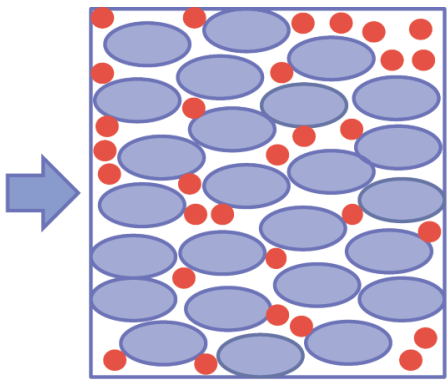

III. La concentration du signal atteint la valeur seuil ou quorum
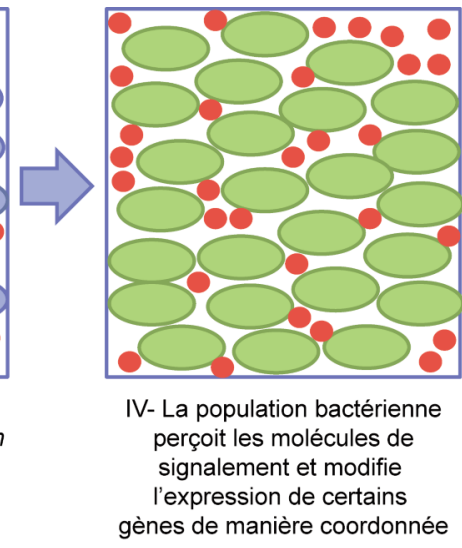
succès pour lutter contre les bactéries pathogènes des plantes (Grandclément et al 2016). Un autre bel exemple de piégeage de molécule de signalement a été rapporté chez Staphylococcus aureus où le peptide de signalement est immobilisé par un anticorps dirigé contre lui. Cette stratégie a donné des résultats convaincants dans un modèle d'infection cutanée chez la souris (Park et al 2007).

c) Perception de la molécule de signalement par un récepteur ou un transporteur en surface de la bactérie

La troisième étape qui peut être ciblée est celle de l'interaction entre la molécule de signalement et son récepteur ou son transporteur à la surface de la bactérie. Là encore un bel exemple est donné chez Staphylococcus aureus où des analogues du peptide de signalement ont été conçus pour inhiber le récepteur de surface (George et al 2008).

D'autres types de travaux utilisent des souches modifiées contenant un gène rapporteur (gène codant une enzyme dont l'activité est facilement quantifiable) sous la dépendance du promoteur du système de «quorum sensing » qui sont utilisées comme biosenseurs (bactéries modifiées utilisées comme outils de détection) pour cribler de manière assez large différentes banques de molécules ou différents échantillons contenant potentiellement des inhibiteurs naturels. Ces approches permettent d'identifier des molécules inhibitrices sans toutefois comprendre à quel niveau du mécanisme de « quorum sensing » elles agissent. Dans ce domaine, des extraits végétaux de différentes origines sont le plus souvent testés et ont montré leur potentiel dans ce domaine (Truchado et al 2015).

\section{3 / Perspectives d'utilisation et réserves techniques}

À notre connaissance, il n'y a pas encore d'application du « quorum quenching " à l'hôpital ou sur le terrain en médecine humaine ou vétérinaire. Les premières applications auront probablement lieu dans le domaine des affections cutanées qui sont les plus accessibles à des molécules ou des extraits inhibiteurs. Pour les infections plus profondes, se pose la question complexe de la galénique (type de présentation du

Figure 6. Représentation schématique des différents niveaux possibles de blocage du mécanisme de régulation de l'expression des gènes par « quorum sensing ».

\section{2- Stabilité et disponibilité de la molécule de signalement}

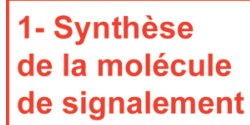

Molécule de signalement

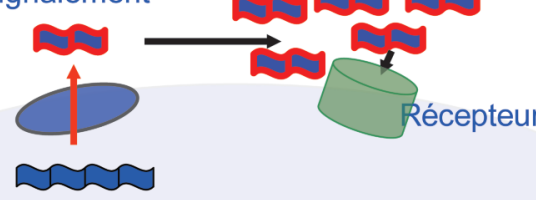

3- Perception de la molécule de signalement

Précurseur de molécule

BACTERIE de signalement

MILIEU EXTRACELLULAIRE

médicament) et de l'accès aux sites d'infection. Dans ce domaine, l'une des pistes de progrès est peut-être l'utilisation de nanoparticules comme vecteurs de « quorum quencheurs », qui a été testée avec succès in vitro (Miller et al 2015). Se pose aussi la question de la nonélimination systématique de la bactérie pathogène et donc aussi de la durée du traitement. Ces stratégies de « quorum quenching » doivent donc sans doute être envisagées en combinaison avec d'autres thérapeutiques.

D'autres approches d'écologie microbienne sont aussi à considérer (Helman et Chernin 2015). Chez les plantes, l'utilisation de bactéries dotées d'activités enzymatiques capables de dégrader les acyl homoserine lactones de bactéries pathogènes des systèmes racinaires et donc de diminuer leur virulence a été testée avec succès. Par exemple, Rhodococcus, agissant comme agent de biocontrôle "quorum quencheur », a été utilisé pour diminuer la virulence de Pectobacterium dans un système de culture hydroponique de pomme de terre. Il est donc possible de modifier les écosystèmes microbiens racinaires en introduisant ou en stimulant des bactéries dotées d'activité « quorum quenching » capables de dégrader ou de capter les molécules de signalement produites par une bactérie pathogène. Ces stratégies de modifications d'écosystèmes microbiens, plus complexes à mettre en œuvre sont cependant intéressantes à explorer à l'avenir.

\section{Conclusion}

À part les alternatives thérapeutiques aux antibiotiques déjà utilisées en pratique, telles que l'homéopathie ou la phytothérapie, d'autres approches sont potentiellement prometteuses pour contrôler les populations bactériennes ou leur virulence, telles que la phagothérapie, l'usage de peptides antimicrobiens ou le "quorum quenching ». Néanmoins différents travaux de recherche puis de développement industriel sont encore nécessaires avant de voir apparaître des produits utilisables en pratique. Par ailleurs, pour la phagothérapie et la phytothérapie, des évolutions réglementaires seraient nécessaires pour faciliter leur commercialisation.

Par ailleurs, il parait illusoire de vouloir remplacer simplement et directement les antibiotiques par ces thérapeutiques alternatives (Gromond 2016b). Intégrer les thérapeutiques alternatives dans l'approche de la santé du troupeau requiert d'avoir engagé une démarche préventive globale en prenant en compte les besoins physiologiques des animaux, en maintenant les conditions d'une bonne immunité innée, en respectant les équilibres microbiens environnementaux (Gromond 2016b), ce qui permet le maintien d'un bon équilibre de la santé du troupeau recherché en Agriculture Biologique (cf. encadré). Dans ces conditions, la thérapeutique ne concerne plus que des cas sporadiques et peut être envisagée avec des solutions alternatives. 
Encadré. Approche de la santé de l'élevage en Agriculture Biologique (Source Denis Fric).

Dans cet article consacré aux alternatives thérapeutiques aux antibiotiques, un encart est consacré à l'approche de la santé en Agriculture Biologique car elle intègre de manière organisée à la fois la prévention des maladies par une approche systémique de la santé et l'utilisation préférentielle de médicaments alternatifs aux antibiotiques quand c'est possible. De fait certains de ces systèmes d'élevage sont en quelque sorte pionniers dans la réduction d'usage des antibiotiques et leur approche pertinente à analyser.

Différents points de vue coexistent sur ce qu'est l'Agriculture Biologique. Dans cet encart, nous ne considérons pas simplement l'Agriculture Biologique comme le respect d'un cahier des charges, avec substitution des intrants chimiques par des intrants biologiques. Nous considérons qu'elle n'est pas une forme d'agriculture parmi d'autres, qu'elle se distingue du modèle intensif de production par une approche différente : " L'Agriculture Biologique est un mode de production, une manière de produire, de respecter et de valoriser l'environnement, de favoriser le bien-être animal, d'être acteur du développement rural, de créer emplois et richesses économiques » (Benoît Canis, préface de Ragot 2001). D'autres approches de l'agriculture partagent certains de ces enjeux, mais les moyens d'y parvenir sont différents ; l'Agriculture Biologique a recours à des pratiques culturales et d'élevage soucieuses du respect des équilibres naturels, notamment en matière de recyclage des matières organiques, de pratiques culturales, d'exclusion des pesticides, etc. (Institut Technique de l'Agriculture Biologique 2016). En matière d'élevage, un des éléments de la démarche de l'Agriculture Biologique est la préservation de la santé collective des animaux. Les aspects sanitaires de l'élevage en Agriculture Biologique vont donc couvrir l'ensemble des particularités d'un système qui peuvent avoir une influence sur la préservation de la santé : sol, cultures, alimentation, conditions d'élevage, contraintes diverses telles qu'éloignement des parcelles ou remboursement d'emprunt, tout en intégrant la vision de l'éleveur sur son exploitation.

\section{Approche globale et équilibre du système d'élevage}

Ces fondements de l'Agriculture Biologique impliquent la nécessité pour celui qui s'intéresse aux aspects sanitaires en Agriculture Biologique d'avoir une approche globale de la ferme. Une des particularités de l'Agriculture Biologique est la diversité de ses systèmes : aucun n'est semblable à un autre et la maîtrise de la santé ne peut de ce fait relever de « recettes » applicables à tous les élevages biologiques. L'objectif est donc de comprendre les " contraintes objectives et les objectifs contraignants » d'une exploitation donnée en mettant en évidence ses facteurs de risque, ses points faibles et en prenant en compte l'ensemble des liens qui unissent étroitement éleveur, sol, plante et animal.

Le système d'élevage doit trouver un équilibre, mais il est souvent en constante évolution car soumis aux aléas du climat, des filières de la commercialisation ou des pratiques de l'éleveur. Cet équilibre n'est donc pas statique mais dynamique (cf. figure 1). L'approche globale de la ferme, à l'occasion d'un " suivi sanitaire », a pour objectif de découvrir les signes et les sources du déséquilibre car, dans la grande majorité des cas, les problèmes sanitaires en Agriculture Biologique ont pour origine un déséquilibre : alimentation mal maîtrisée, bâtiment mal adapté ou techniques d'élevage inappropriées. Ceci est aussi vrai en agriculture conventionnelle ; néanmoins l'approche en Agriculture biologique met l'accent sur la correction d'ensemble de ces déséquilibres dans une approche systémique, afin d'éviter le recours aux médicaments.

Figure. Schéma représentant la réaction de l'animal confronté à des déséquilibres du système d'élevage.

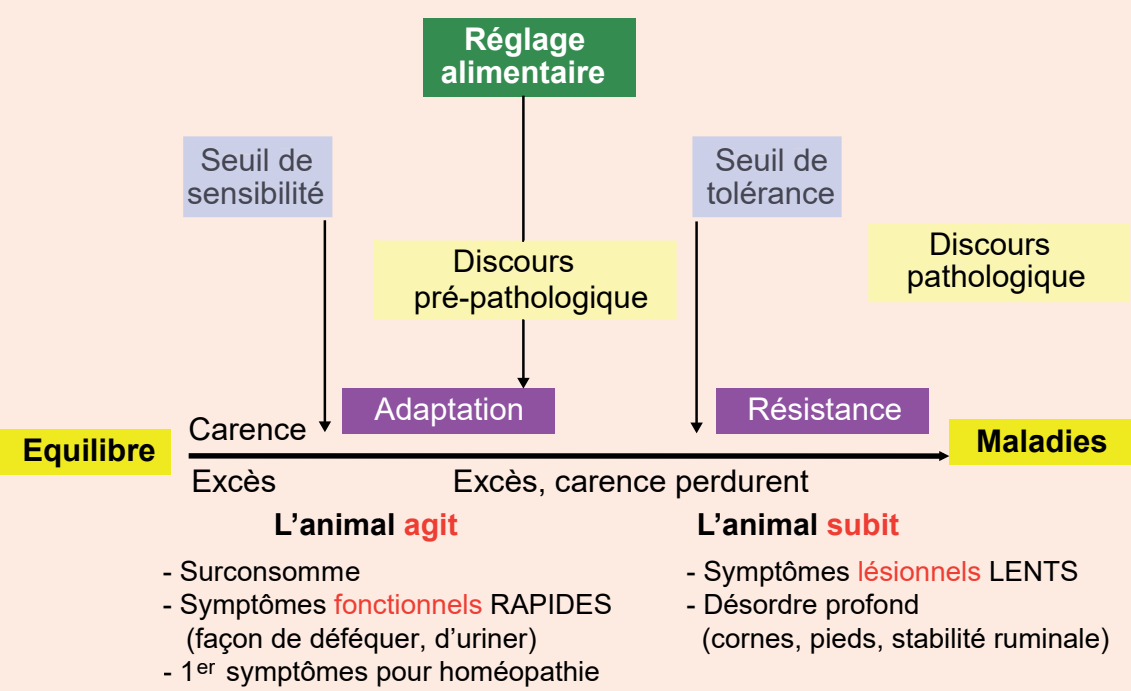

\section{Observation du troupeau}

L'animal est le premier à extérioriser les déséquilibres du système (cf. fgure); il est l'élément le plus réactif par son « discours pathologique » (impact clinique du déséquilibre sur la santé quand le seuil de tolérance a été dépassé) et, avant cette phase, par les signes d'adaptation ou de tolérance qui précèdent l'atteinte clinique : son " discours pré-pathologique ». Ce discours pré-pathologique peut s'exprimer de diverses manières, notamment par des signes alimentaires (mouvements de poils, matière 
sébacée, mis en exergue par la méthode Obsalim (Giboudeau 2016), des signes comportementaux (concurrence alimentaire, rythmes d'un lot ou d'un troupeau....) ou physiques (toux, vaches qui défèquent en salle de traite...).

La compréhension du fonctionnement de la ferme et son approche globale reposent donc avant tout sur l'observation : savoir observer ses animaux et prendre le temps de les observer est un principe de base pour tous les éleveurs, en particulier en Agriculture Biologique. Par ailleurs, pour l'éleveur qui a « le nez dans le guidon ", il peut être difficile de prendre du recul et l'analyse par un œil extérieur à la ferme est souvent un besoin, d'où l'apport bénéfique de l'œil extérieur de groupes d'éleveurs, du technicien ou du vétérinaire. Sur la base de l'analyse de ces observations, l'enjeu est de mettre en œuvre des améliorations afin d'élargir au maximum la zone de bien-être et d'équilibre de l'animal pour rendre le système moins sensible aux diverses agressions de type aléas climatiques, mauvaises récoltes, augmentation du chargement par report de ventes etc.

\section{Approche des problèmes sanitaires}

Ainsi, la visite lors d'un suivi sanitaire comprend deux aspects :

- Proposer des mesures préventives pour corriger les facteurs de risque identifiés lors de l'observation et de l'analyse de l'ensemble des signes exprimés par les animaux. Ces mesures doivent être compatibles avec les objectifs et les possibilités de l'éleveur. Le " discours pré-pathologique » des animaux permet de hiérarchiser les facteurs et choisir ceux qui ont le plus d'impact sur l'élevage.

- Apporter en cas de besoin des solutions thérapeutiques allant dans le sens de l'éthique et du Cahier des Charges de l'Agriculture Biologique : donner la priorité aux médecines naturelles. L'homéopathie et la phytothérapie, même si elles ne sont pas les seules, ont leur place dans le choix d'alternatives thérapeutiques à l'allopathie utilisant des produits chimiques de synthèse.

Pour simplifier, ceci revient à dire que toutes les pathologies collectives relèvent de l'observation des signes pré-pathologiques et de la correction des facteurs de risque, qui peuvent dans certains cas être couplées avec une thérapeutique de type homéopathie ou médecine naturelle ; et que toutes les pathologies individuelles (accidents cliniques) relèvent des médecines naturelles, par exemple de l'homéopathie qui est basée sur l'observation des signes exprimés par l'animal face à son déséquilibre.

Pour le vétérinaire, cette approche est néanmoins difficile en raison du manque de références : l'analyse des pratiques et des savoir-faire des éleveurs pour la gestion de la santé de leurs animaux est encore à découvrir. Les données sur les comportements des animaux sont peu documentées ; à titre d'exemple quelle est l'influence sur la santé au pâturage de prairies à flores complexes par rapport au pâturage « plat unique »?

\section{Questions de recherche}

Les éleveurs sont dépositaires de savoir-faire et d'observations sur leur ferme et il est important de recueillir, de diffuser et de faire échanger les éleveurs sur leurs pratiques. La recherche en Agriculture Biologique aurait beaucoup à apprendre des éleveurs. Plutôt que de poser la question « comment faire pour respecter le nombre de traitements chimiques autorisé par le Cahier des Charges ? " une démarche plus proactive consiste à se demander " comment augmenter les défenses immunitaires de mes animaux, comment mieux équilibrer leur alimentation ? ».

De nombreuses années d'exercice de l'auteur l'ont amené à visiter des fermes en Agriculture Biologique dans des régions diverses et des types de production différents : bovins viande, bovins lait, ovins, caprins avec des ateliers multiples ou spécialisés. Certaines de ces fermes ont su trouver un équilibre presque parfait dans lequel les problèmes sanitaires se résument à des " accidents " : maîtrise de l'alimentation, plus aucun traitement antiparasitaire, bien-être des animaux (et de l'éleveur) visible. Examiner pourquoi certains systèmes ont une telle capacité de résilience pourrait donner des pistes sur les pratiques favorisant la résistance aux déséquilibres, donc aux pathologies. La difficulté de cette recherche réside dans la nécessité d'aborder ces questions à l'échelle du système car décomposer l'approche en analysant séparément chacune de ses parties ne permet pas de répondre à la question de manière pertinente. Au-delà de l'Agriculture Biologique, ces travaux devraient permettre d'acquérir des connaissances généralisables aux autres systèmes d'élevage.

\section{Références}

Ackermann H.W., 2003. Bacteriophage observations and evolution. Res. Microbiol., 154, $245-251$.

Anses, 2016. Avis de l'Anses. Évaluation des demandes d'autorisation de mise sur le marché des médicaments vétérinaires à base de plante. Rapport 119pp https://www.anses.fr/fr/system/ files/MV2014SA0081Ra.pdf Consulté le 20 juillet 2016
Ansoud C., 2016. Utilisation de l'homéopathie dans un cas de diarrhée chez deux veaux d'une semaine. In : Soigner autrement : trouver l'équilibre pour produire mieux. Le point vétérinaire Numéro spécial, 47, 104-107.

Bulfon C., Volpatti D., Galeotti M., 2015 Current research on the use of plant-derived products in farmed fish. Aquacult. Res., 46, 513-551.
Carlet J., Le Coz P., 2015. Tous ensemble, sauvons les antibiotiques. Rapport du groupe de travail pour la préservation des antibiotiques. Juin 2015, 150 pp. http://social-sante.gouv.fr/ IMG/pdf/rapport antibiotiques.pdf Consulté le 19 juillet 2016.

Carlton R.M., 1999. Phage therapy: past history and future prospects. Arch. Immunol. Ther. Exp., (Warsz), 47, 267-274. 
De Lange C., Pluske J., Gong J., Nyachoti C., 2010. Strategic use of feed ingredients and feed additives to stimulate gut health and development in young pigs. Livest. Sci., 134, 124-134.

Diaz Carrasco J.M., Redondo L.M., Redondo E. A., Dominguez J.E., Chacana A.P., Fernandez Miyakawa M.E., 2016. Use of plant extracts as an effective manner to control clostridium perfringens induced necrotic enteritis in poultry. Biomed Rese. Int., Article ID $3278359,15 \mathrm{p}$

Donadio S., Maffioli S., Monciardini P., Sosio M., Jabes D., 2010. Antibiotic discovery in the twenty-first century: current trends and future perspectives. J. Antibiot., (Tokyo) 63, 423-430.

Dordain C., 2016a. Apports de la phytothérapie chez les bovins. In : Soigner autrement : trouver l'équilibre pour produire mieux. Le point vétérinaire Numéro spécial, 47, 68-75.

Dordain C., 2016b. Cas concrets d'utilisation de l'homéopathie en pratique rurale quotidienne. In : Numéro spécial, Soigner autrement : trouver l'équilibre pour produire mieux. Le point vétérinaire, $47,58-67$.

Enault F., Briet A., Bouteille L., Roux S., Sullivan M.B., Petit M.A., 2016. Phages rarely encode antibiotic resistance genes: a cautionary tale for virome analyses. ISME J, 11, 237-247.

European Commission, 2011. Action Plan against the rising threats from antimicrobial resistance. Rapport 15pp. Consultable à: http://ec.europa.eu/ dgs/health food-safety/docs/communication amr_2011_748_en.pdf

Filliat C., Souvestre M., 2016. Utilisation de l'homéopathie et de la phytothérapie en médecine collective : résultats obtenus en élevage de volailles. In : Numéro spécial, Soigner autrement : trouver l'équilibre pour produire mieux. Le point vétérinaire, $47,90-95$.

George E.A., Novick R.P., Muir T.W., 2008. Cyclic peptide inhibitors of staphylococcal virulence prepared by Fmoc-based thiolactone peptide synthesis. J. American Chem. Soc., 130, 4914-4924.

Grandclément C., Tannieres M., Morera S., Dessaux Y., 2016. Quorum quenching : role in nature and applied developments. FEMS Microbiol. Rev. 40, 86-116.

Gromond G., 2016a. Évaluation et comparaison des différentes thérapeutiques alternatives en élevage. In : Numéro spécial, Soigner autrement : trouver l'équilibre pour produire mieux. Le point vétérinaire, $47,84-89$.

Gromond G., 2016b. Santé animale et stratégie alternative en techniques d'élevage. In : Numéro spécial, Soigner autrement : trouver l'équilibre pour produire mieux. Le point vétérinaire, 47 , 108-113.

Hancock R.E., Haney E.F., Gill E.E., 2016. The immunology of host defence peptides: beyond antimicrobial activity. Nat. Rev. Immunol., 16, 321-334.

Helman Y., Chernin L., 2015. Silencing the mob: disrupting quorum sensing as a means to fight plant disease. Mol. Plant Pathol., 16, 316-329.

Inal J.M., 2003. Phage therapy: a reappraisal of bacteriophages as antibiotics. Arch. Immunol. Ther. Exp., (Warsz), 51, 237-244.
Institut Technique de l'Agriculture Biologique, 2016. Institut Technique de l'Agriculture Biologique. Généralités sur l'AB. http://www.itab. asso.fr/abinfo/AB.php

Issautier M.N., 2016. Fondements de 1'homéopathie et principes d'utilisation en élevage bovin. In : Numéro spécial, Soigner autrement : trouver l'équilibre pour produire mieux. Le point vétérinaire, 47, 51-57.

Journal Officiel de la République Française, 2013. Décret n²013-752 du 16 août 2013 portant sur diverses dispositions relatives aux médicaments vétérinaires et aux établissements pharmaceutiques vétérinaires https://www legifrance.gouv.fr/affichTexte.do? cidTexte $=\mathrm{JO}$ RFTEXT000027845643\&categorieLien $=\mathrm{id}$ Consulté le 20 juillet 2016.

Journal Officiel de l'Union Européenne, 2009. Règlement $(\mathrm{CE}) \mathrm{n}^{\circ} 470 / 2009$ établissant des procédures communautaires pour la fixation des LMR des substances pharmacologiquemen actives dans les aliments d'origine animale http://ec.europa.eu/health/files/eudralex/vol $5 / \mathrm{reg} 2009-470 / \mathrm{reg} 470 \quad 2009$ fr.pdf Consulté le 20 juillet 2016 .

Journal Officiel de 1'Union Européenne, 2009. Règlement (UE) n³7/2010 relatif aux substances pharmacologiquement actives et à leu classification en ce qui concerne les limites maximales de résidus (LMR) dans les aliment d'origine animale http://eur-lex.europa.eu/ LexUriServ/LexUriServ.do?uri=OJ:L:2010:01 5:0001:0072:FR:PDF Consulté le 20 juillet 2016.

Lecat A., 2016. Acupuncture vétérinaire concepts. In : Numéro spécial, Soigner autrement : trouver l'équilibre pour produire mieux. Le point vétérinaire, $47,16-21$.

Le point vétérinaire 2016. Soigner autrement trouver l'équilibre pour produire mieux. Le point vétérinaire, Numéro spécial, 47, 140p.

Lidor O., Al-Quntar A., Pesci E.C., Steinberg D. 2015. Mechanistic analysis of a synthetic inhibitor of Pseudomonas aeruginosa Las quorum-sensing signal synthase. Scientific Reports, 5, article 16569

Loc-Carrillo C., Abedon S.T., 2011. Pros and cons of phage therapy. Bacteriophage, 1, 111-114

Mauvisseau T., 2016. Le phytogramme : un nouvel outil utilisable en élevage. In : Numéro spécial, Soigner autrement : trouver l'équilibre pour produire mieux. Le point vétérinaire, 47 , $76-83$

Mayer M., Vogl C.R., Amorena M., Hamburger M., Walkenhorst M., 2014. Treatment of organic livestock with medicinal plants: A systematic review of European ethnoveterinary research. Forschende Komplementarmedizin 21, 375-386.

Miller K. P., Wang L., Chen Y.P., Pellechia P.J., Benicewicz B.C., Decho A.W., 2015. Engineering nanoparticules to silence bacterial communication. Front. Microbiol., 6, 189

Ministère de l'agriculture 2012. Plan EcoAntibio 2017. Plan national de réduction des risques d'antibiorésistance en médecine vétérinaire. Rapport 32pp http://agriculture gouv.fr/ministere/plan-national-de-reduction-des-risquesdantibioresistance-en-medecine-veterinaire consulté le 20 juillet 2016.

Monnet V., Juillard V., Gardan R., 2016 Peptide conversations in Gram-positive bacteria Crit. Rev. Microbiol. 42, 339-351.
Newman D.J., Cragg G.M., 2012. Natural products as sources of new drugs over the 30 years from 1981 to 2010. J. Nat. Prod., 75, 311-335.

OMS, 2015. Document A68/20 de la $68^{\text {èm }}$ Assemblée mondiale de la santé, Résistance aux antimicrobiens - Projet de plan d'action mondial pour combattre la résistance aux antimicrobiens. Rapport 20pp. http://apps.who.int/ $\mathrm{gb} / \mathrm{ebwha/pdf}$ files/WHA68/A68 20-fr.pdf consulté le 20 juillet 2016.

Park J., Jagasia R., Kaufmann G.F., 2007. Infection control by antibody disruption of bacterial quorum sensing signaling. Chem. Biol., 14, 1119-1127.

Pires D.P., Oliveira H., Melo L.D., Sillankorva S., Azeredo J., 2016. Bacteriophage-encoded depolymerases: their diversity and biotechnological applications. Appl. Microbiol. Biotechnol., 100, 2141-2151.

Pirnay J.P., De Vos D., Verbeken G., Merabishvili M., Chanishvili N., Vaneechoutte M., Zizi M., Laire G., Lavigne R., Huys I., Van den Mooter G., Buckling A., Debarbieux L., Pouillot F., Azeredo J., Kutter E., Dublanchet A., Gorski A., Adamia R., 2011. The phage therapy paradigm: pret-a-porter or sur-mesure? Pharm. Res., 28, 934-937.

Pirnay J.P., Blasdel B.G., Bretaudeau L., Buckling A., Chanishvili N., Clark J.R., CorteReal S., Debarbieux L., Dublanchet A., De Vos D., Gabard J., Garcia M., Goderdzishvili M., Gorski A., Hardcastle J., Huys I., Kutter E., Lavigne R., Merabishvili M., Olchawa E., Parikka K.J., Patey O., Pouilot F., Resch G., Rohde C., Scheres J., Skurnik M., Vaneechoutte M., Van Parys L., Verbeken G., Zizi M., Van den Eede G., 2015. Quality and safety requirements for sustainable phage therapy products. Pharm. Res., 32, 2173-2179.

Prado Montes de Oca E., 2013. Antimicrobial peptide elicitors: new hope for the post-antibiotic era. Innate immunity, 19, 227-241.

Prevel R., Dufour N., 2016. Potential use of bacteriophages in modern infectiology. Rev. Med. Interne, 10, 657-660

Projan SJ, Shlaes D.M., 2004. Antibacterial drug discovery: is it all downhill from here? Clin. Microbiol. Infect., 10, 18-22.

Ragot M., 2001. Conversion à l'agriculture biologique : le cas de la production laitière. Educagri éditions, Dijon, France, 355p.

Sulakvelidze A., Alavidze Z., Glenn Morris Jr. J., 2001. Bacteriophage therapy. Antimicrob. Agents Chemother., 45, 649-659.

Truchado P., Larrosa M., Castro-Ibanez I., Allende A., 2015. Plant foods extracts and phytochemicals: their role as quorum sensing inhibitors. Trends Food Sci. Technol., 43, 189-204.

WHO, FAO, OIE 2010. Unite in Antimicrobial Resistance the fight against antimicrobial resistance. Rapport 2pp. http://www.oie.int/fileadmin Home/eng/Media Center/docs/pdf/FAO OIE WHO AMRfactsheet.pdf consulté le 20 juillet 2016

Zahar J.R., Lesprit P., 2014. Management of multidrug resistant bacterial endemic. Medecine et maladies infectieuses. 44, 405-411.

Zasloff M., 2002. Antimicrobial peptides of multicellular organisms. Nature, 415, 389-395. 


\title{
Résumé
}

Les principes, contraintes et questions de recherche relatifs à plusieurs perspectives d'alternatives thérapeutiques aux antibiotiques sont présentés. Différentes approches telles que la phytothérapie et la phagothérapie, basée sur l'activité lytique des phages infectant les bactéries, sont anciennes mais leur utilisation pratique nécessiterait des recherches complémentaires en matière de qualité, efficacité et sécurité ainsi que des évolutions réglementaires. D'autres approches sont plus récentes et requièrent de poursuivre des travaux de recherche avant d'envisager leur application sur le terrain. C'est le cas notamment des peptides de défense de l'hôte, molécules de l'immunité innée produites essentiellement par les cellules épithéliales et phagocytes, qui ont une activité antimicrobienne à large spectre et un pouvoir de régulation de l'immunité. Il y a peu de développement industriel lié à des difficultés techniques, malgré le grand nombre de peptides candidats. Une autre approche concerne le « quorum sensing », lié à la sécrétion de molécules de signalement par les bactéries, qui permet des changements coordonnés chez les bactéries présentes au-delà d'un certain seuil, notamment la production de facteurs de virulence ou de biofilms. Le blocage de ces mécanismes, ou « quorum quenching », pourrait permettre de réguler ces propriétés.

\begin{abstract}
Prospects for therapeutic alternatives to antimicrobials on farms

Principles, constraints and research questions related to several perspectives of therapeutic alternatives to antimicrobials are presented. Different approaches are not new such as phytotherapy and phagotherapy that relies on the lytic properties of phages infecting bacteria, but their use in practice would require further research on quality, efficacy and security of the products, as well as regulatory developments. Other approaches are more recent and require further research before considering their application in the field. This is the case for host defence peptides, molecules of innate immunity that are mainly produced by epithelial cells and phagocytes; they have large spectrum antimicrobial properties and immunity regulation capacity. There are few industrial developments due to technical problems despite a high number of candidates. Another approach concerns quorum sensing, based on signalling molecules produced by bacteria, that allows coordinated changes of the bacteria properties above a certain threshold, such as producing virulent factors or biofilms. Quorum quenching might allow regulating these properties.
\end{abstract}

DUCROT C., FRIC D., LALMANACH A.-C., MONNET V., SANDERS P., SCHOULER C., 2017. Perspectives d'alternatives thérapeutiques antimicrobiennes aux antibiotiques en élevage. INRA Prod. Anim., 30, 77-88. 
